\title{
Clinico-pathological characteristics and outcome of upper urinary tract urothelial carcinoma presenting to a tertiary care urology unit in Sri Lanka
}

\author{
U Jayarajah'1, M H Fernando1, K B Herath'1, S N Kuruppu', U L Wickramanayaka1, I U Fernando1, \\ V C de Silva ${ }^{2}$, S A S Goonewardena ${ }^{1}$
}

(Index words: Upper urinary tract, urothelial carcinoma, clinicopathological characteristics, Sri Lanka)

\begin{abstract}
Introduction Upper urinary tract urothelial cancers account for $5 \%$ of urothelial tumours. In the West, the majority affect the pelvicalyceal system, with pyelocalyceal to ureteric ratio of $3: 1$. This study aims to describe the clinico-pathological features and outcome of upper urinary tract urothelial cancer treated surgically in a tertiary care unit in Sri Lanka.
\end{abstract}

Methods A retrospective analysis of all patients who underwent nephroureterectomy for upper urinary tract urothelial cancer at the Urology Unit at National Hospital of Sri Lanka between January1997 and December 2016 was carried out.

Results There were 43 patients. Male: female $=1.87$. Median age was 65 years (range:42-83). Macroscopic haematuria was the commonest presentation $(n=29$; $67.4 \%$ ). Median duration of symptoms was 3 months (range $0.5-6)$. In the majority $(n=20 ; 46.5 \%)$ the tumour was confined to the ureter. Thirty-three $(75.6 \%)$ were papillary tumours. Twenty-one had non-muscle invasive tumours (pTa: $n=6(14 \%), p T 1: n=15(34.9 \%)$ and others had invasive cancers (pT2: $n=11$ (25.6\%), pT3: $n=7$ $(16.3 \%)$ and pT4: $n=4(9.3 \%))$. Majority were low grade tumours $(n=23 ; 53.5 \%)$. Twelve $(27.9 \%)$ had preceding urothelial bladder cancer. Nineteen $(44.2 \%)$ were lost to follow up after surgery. Median follow up duration of the rest was 40 months (range: 4-224 months). Of them, $9(20.9 \%)$ developed metachronous bladder tumours. Nine had recurrence free survival of $\geq 5$ years and 15 had overall survival of $\geq 5$ years. Of them, 4 patients survived $\geq 10$ years. Older age $(p=0.015)$ and presence of necrosis $(p=0.05)$ were the only clinico-pathological parameters predictive of tumour recurrence.

Conclusion A relatively higher number females and high number of ureteric tumours were noted compared to similar studies from Asia.
Ceylon Medical Journal 2018; 63: 129-132

DOI: http://doi.org/10.4038/cmj.v63i3.8717

\section{Introduction}

Upper urinary tract urothelial carcinomas are rare. They account for 5\% of all urothelial tumours and $10 \%$ of all renal tumours [1]. Upper urinary tract urothelial cancers include those that occur in the renal pelvis and the ureter. These tumours arise from the transitional epithelium, similar to urothelial bladder cancers. Of those, primary tumours in the renal pelvis are more common and occur three to four times more commonly than primary ureteric tumours [2].

Although, minimally invasive endoscopic procedures show favourable survival rates in selected patients with small, low-grade tumours, these approaches are generally not effective because of the associated high recurrence rates [3]. Therefore, nephroureterectomy with excision of a cuff of bladder remains the standard of care for upper urinary tract urothelial cancer $[3,4]$. However, these tumours are notorious for their aggressive behaviour and local failure rates are high despite radical surgery [1]. An important aspect in the management of upper urinary tract urothelial cancer is follow up for recurrence because of the risk of developing urothelial cancers in the remaining transitional cell epithelium [2]. Due to its low prevalence, the clinical and pathological characteristics of upper urinary tract urothelial cancer are not well known especially among South Asians [5]. Although there are data on urothelial and non-urothelial bladder cancer in Sri Lanka there are no publications regarding the patterns of upper urinary tract urothelial cancer in Sri Lanka [6,7]. In the present study, we describe the main clinico-pathological characteristics of patients with upper urinary tract

${ }^{1}$ Department of Urology, National Hospital of Sri Lanka, Colombo, ${ }^{2}$ Department of Pathology, Faculty of Medicine, University of Colombo, Sri Lanka.

Correspondence: UJ, e-mail: <umeshe.jaya@gmail.com>. Received 04 June 2018 and revised version accepted 8 August 2018.

(C) (i) This is an open-access article distributed under the terms of the Creative Commons Attribution License, which permits unrestricted use, distribution, and reproduction in any medium, provided the original author and source are credited. 
urothelial cancer who were treated with radical surgery, and the occurrence of synchronous and metachronous bladder tumours.

\section{Methodology}

A retrospective analysis was carried out on all patients diagnosed with upper urinary tract urothelial cancer at the Urology Unit, National Hospital of Sri Lanka between January 1997 and December 2016. The clinicopathological variables were recorded.

All patients underwent nephroureterectomy and bladder cuff excision using standard procedure. Cystoscopy was done on every patient to look for concomitant bladder tumours (synchronous tumours). Data were collected at the time of initial hospitalisation and during clinic visits. Data was recorded on a separate file for each patient, rendering accurate documentation and easy retrieval of details. The demographic details, clinical presentation, method of diagnosis and histopathological characteristics were analysed. Pathological grading was assessed according to the 1973 World Health Organisation (WHO) classification in patients from 1997- 2006 and the WHO and International Society of Urological Pathology (ISUP) classification 2004 in patients from 20072016. WHO 2004 classification which was used in the first half of the study has three different grades of anaplasia (G1, G2, and G3 or high grade) while the 2004 ISUP/WHO classification which was used in the latter half has only two grades of anaplasia (low and high grades) [8]. In our analysis, we considered $\mathrm{G} 1$ and G2 as low grade and G3 as high grade tumours.

Those with muscle invasive disease were referred to the oncologist for adjuvant chemoradiation. Thereafter, patients were followed-up with check cystoscopy every 3 months during the first year and thereafter annually. Annual ultrasound scan was done to assess the upper urinary tract of the contralateral side. Follow up details including recurrence, recurrence free survival, and overall survival were calculated.

Data were analysed using SPSS software version 17. Statistical analysis was done using non-parametric tests such as Mann-Whitney U test and Chi-square test. Kaplan Meir survival analysis was done to derive recurrence-free survival. Approval for the study was obtained from the Ethical Review Committee of National Hospital of Sri Lanka.

\section{Results}

Sample included 43 patients. The majority were males $(n=28 ; 65.1 \%)$ with a male to female ratio of 1.87 . Median age was 65 years (range 42-83). Macroscopic haematuria was the commonest clinical presentation $(n=29 ; 67.4 \%)$ followed by loin pain $(n=13 ; 30.2 \%)$. Twelve $(30 \%)$ had a history of passage of clots and two patients $(4.7 \%)$ passed fleshy particles. The tumour was incidentally detected in
$9(20.9 \%)$ patients. The median duration of symptoms was 3 months (range 0.5-6).

Left sided tumours were present in 24 (55.8\%). In 20 (46.5\%), tumour was confined to the ureter and in 13 $(30.2 \%)$ to the renal pelvis. In $10(23.3 \%)$, the tumours were found in both ureter and renal pelvis.

The majority $(\mathrm{n}=33 ; 75.6 \%)$ were papillary tumours, 6 (14\%) were solid tumours and $4(23.3 \%)$ were mixed tumours (both papillary and solid components). Ulceration was seen in two $(4.7 \%)$. Majority $(n=35 ; 81.4 \%)$ were solitary tumours. Non muscle invasive tumours were seen in 21 (pTa: $n=6 ; 14 \%, p T 1: n=15 ; 34.9 \%$ ) and the rest were invasive carcinomas (pT2: $\mathrm{n}=11 ; 25.6 \%, \mathrm{pT} 3: \mathrm{n}=7 ; 16.3 \%$ and pT4: $n=4 ; 9.3 \%)$. Twenty three $(53.5 \%)$ were low grade tumours and the rest $(n=20 ; 46.5 \%)$ were high grade. One tumour had $50 \%$ squamous differentiation. Lymphovascular invasion was seen in 7 (16.3\%).

There was a history of urothelial bladder cancer in 12 (27.9\%). Synchronous bladder tumours (concurrent bladder tumours at the time of initial diagnosis) were present in 4 (9.3\%). Nineteen $(44.2 \%)$ were lost to follow up after surgery. The median follow up duration of the rest $(n=24)$ was 40 months (Inter quartile range 5.4-69 months). Of those who were followed up $9(20.9 \%)$ developed metachronous bladder tumours (bladder tumours detected during follow up check cystoscopy). Eight developed these tumours within one year of surgery and in the other patient the recurrence was detected after seven years during annual check cystoscopy. Fifteen patients $(34.9 \%)$ had no recurrence. Of those with recurrence, 4 had small papillary recurrences which were treated with transurethral fulguration. Others underwent transurethral resection of bladder tumour (TURBT) and the histology showed pTa high grade in 2, pT1 low grade in 2 and pT1 high grade in one patient.

Despite the limitation of follow up, $9(37.5 \%)$ had recurrence free survival of 5 years or more and while 4 $(16.7 \%)$ had recurrence free survival of more than 10 years (Figure 1). Fifteen patients $(62.5 \%)$ survived for more than 5 years and of them, 4 survived $(16.7 \%)$ beyond 10 years.

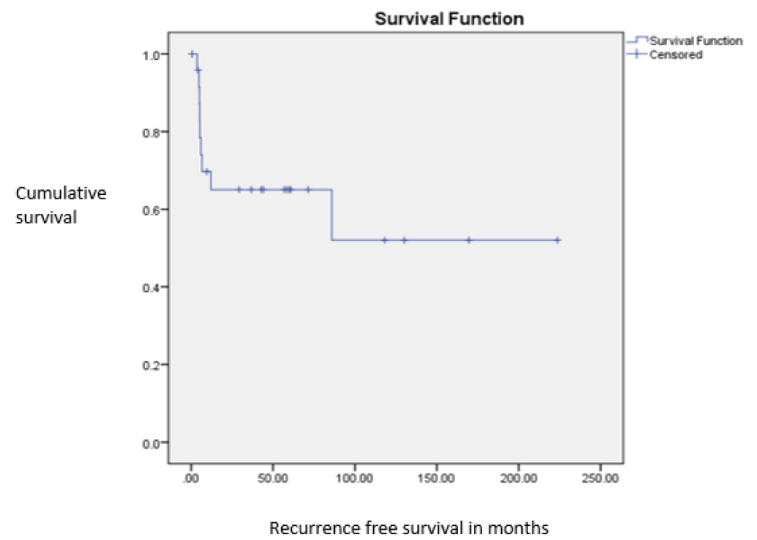

Figure 1. Kaplan Meir survival plot of recurrence free survival. 
Older age $(\mathrm{p}=0.015)$ and presence of necrosis (OR $3.1 ; 95 \%$ CI $1.7-5.8 ; \mathrm{p}=0.05)$ were the only clinicopathological parameters which was associated with tumour recurrence. Other factors such as sex, clinical presentation, tumour grade and tumour stage did not have a significant association with tumour recurrence.

\section{Discussion}

This study presents the clinical and histopatholoigical characteristics of upper urinary tract urothelial cancer in a cohort of patients. Left sided tumours were more common (55.8\%). Twenty $(46.5 \%)$ had a tumour confined to the ureter, $13(30.2 \%)$ to the renal pelvis and the rest had a tumour involving the renal pelvis and ureter. Twenty two (51.2\%) had muscle invasive disease and 20 (46.5\%) had high grade tumours.

Most evidence on upper urinary tract urothelial cancer are from developed countries and there are only few studies from South Asia [5]. Upper urinary tract urothelial cancer occurs at a mean age of 60 - 70 years. Our study had similar findings [5, 9-11]. A male preponderance has been reported in the Western literature with a male to female ratio of $3: 1[9,10]$. A study from India reported a higher male preponderance (male: female $=7: 1$ ) which was attributed to low prevalence of smoking in females, a major risk factor in urothelial cancers [5]. However, in our study, although the disease was commoner in males, the male to female ratio was only 1.87:1. This indicates a considerably higher proportion of females compared to findings from India and the Western countries. This is quite unusual because of the low prevalence of smoking among females in Sri Lanka and India. A study from Sri Lanka on urothelial bladder cancers by Goonewardena et al. shows high male preponderance of 6:1 [6]. Therefore, the reason for the unusually high proportion (35\%) of females developing upper urinary tract urothelial cancer needs to be further evaluated.

In the present study, $51 \%$ of tumours were muscle invasive. The staging of upper urinary tract urothelial cancers differ in different studies, with reported incidence of muscle invasive cancer of $40-56 \%$ [9, 10, 12]. A recent study from India showed that $88 \%$ of upper urinary tract urothelial cancer were muscle invasive. This is a considerably high rate. Inadequate attention to microscopic haematuria and very late presentation has been postulated as possible reasons for the high proportion of muscle invasive disease [5].

In the present study $46.5 \%$ were high grade tumours. A study from India reported prevalence of high grade tumours of about $66 \%$. Other studies report prevalence rates from $36-58 \%[10,12]$. The high prevalence of muscle invasive disease and high grade tumours is clinically significant as they indicate poor prognosis [5].

In Western countries tumours of the renal pelvis are reported to be 3 to 4 times commoner than ureteric tumours
[2]. In the Indian study 29 out of 36 tumours were confined to the renal pelvis [5]. However, in the present study, majority of patients $(46.5 \%)$ had tumours confined to the ureter, $30.2 \%$ to the renal pelvis and the rest had tumour in renal pelvis and ureter. Thus, our findings show a different pattern compared to other studies conducted in South Asia and the West.

In the present study, $20.9 \%$ of the followed up patients developed metachronous bladder tumours. Eight developed within one year of surgery and recurrence was detected in the other patient after 7 years during annual check cystoscopy. The recurrence rate in the present study is comparable to those from India and the West [5]. It is important to note that all patients were asymptomatic when the metachronous tumour was detected and one patient developed recurrence after 7 years. This highlights the importance of rigorous long term follow up in these patients. All recurrences detected in patients who were followed up were non-muscle invasive tumours, which may be due to early detection through regular check cystoscopy.

We found that, older age and presence of necrosis were the only factors associated with tumour recurrence. A recent study of 36 patients from India, identified presence of tumour necrosis as the only risk factor for overall survival [5]. We may have failed to detect other risk factors for recurrence due to the small sample size.

A significant proportion of patients (44\%) was lost to follow up after initial surgery. The median duration of follow up was only 40 months. This is a reflection of the large catchment population of the institution (over 8 million) and the long distances (over 100-200 km) travelled by some patients to come to hospital. We can assume that some of the patients who were lost to follow up had not developed recurrent episodes of visible haematuria, a symptom of neglected urothelial malignancy, which would have compelled them to report back to the same unit [13]. Other reasons for drop outs are financial and sociocultural as well as the stigma related to cancer in South Asia which could result in defaulting treatment.

In our study, rate of five year recurrence-free was $37.5 \%$ and overall survival was $62.5 \%$. In a similar study from India, rate of five-year recurrence-free was 36.3\% and cancer specific survival was $26 \% \pm 5.1 \%$ [5]. However, studies from the West report much better survival. Berger et al. reported five-year cancer specific survival of around $72 \%$ [10]. Margulis et al. reported five-year recurrencefree rate of $69 \%$ and cancer specific survival of $73 \%$ [14]. The reason for reduced survival may be inadequate follow up or presentation with advanced disease.

Our study has several limitations which include the small sample size and limited follow up. The small sample size despite a 20 year period may be due to the rarity of the disease. A considerable number of patients did not present for check cystoscopy after surgery and were lost to follow up. 


\section{Conclusion}

This study identified several patterns that are different to those from Indian and Western countries which include a relatively high prevalence in females and higher occurrence of ureteric tumours compared to renal pelvic tumours.

\section{Conflicts of Interest}

The authors declare that there is no conflicts of interest.

\section{References}

1. Park S, Hong B, Kim CS, Ahn H. The impact of tumor location on prognosis of transitional cell carcinoma of the upper urinary tract. J Urol 2004; 171: 621-5.

2. Kirkali Z, Tuzel E. Transitional cell carcinoma of the ureter and renal pelvis. Critical Reviews in Oncology/Hematology. 47: $155-69$

3. Zigeuner R, Pummer K. Urothelial carcinoma of the upper urinary tract: surgical approach and prognostic factors. Eur Urol 2008; 53: 720-31.

4. Oosterlinck W, Solsona E, van der Meijden AP, et al. EAU guidelines on diagnosis and treatment of upper urinary tract transitional cell carcinoma. Eur Urol 2004; 46: 147-54.

5. Radhakrishnan S, Aga P, Jain M, Srivastava A, Kapoor R, Mandhani A. Clinicopathological spectrum and the outcome of treatment of upper tract transitional cell carcinoma. Indian J Urol 2012; 28: 174-8.

6. Goonewardena SA, De Silva WA, De Silva MV. Bladder cancer in Sri Lanka: experience from a tertiary referral center. Int J Urol 2004; 11: 969-72.

7. Jayarajah U, Herath KB, Fernando MH, et al. A clinicopathological study of non-urothelial bladder cancers in a cohort of patients from a tertiary care urology unit in Sri Lanka. Ceylon Med J 2018; 63(2): 92-4.

8. Cheng L, MacLennan GT, Lopez-Beltran A. Histologic grading of urothelial carcinoma: a reappraisal. Hum Pathol 2012; 43: 2097-108.

9. Fernandez MI, Shariat SF, Margulis V, et al. Evidence-based sex-related outcomes after radical nephroureterectomy for upper tract urothelial carcinoma: results of large multicenter study. Urology 2009; 73: 142-6.

10. Berger A, Haber GP, Kamoi K, et al. Laparoscopic radical nephroureterectomy for upper tract transitional cell carcinoma: oncological outcomes at 7 years. J Urol 2008; 180: $849-54$

11. Park S, Hong B, Kim C-s, Ahn H. The impact of tumor location on prognosis of transitional cell carcinoma of the upper urinary tract. J Urol 2004; 171: 621-5.

12. Li WM, Li CC, Ke HL, Wu WJ, Huang CN, Huang CH. The prognostic predictors of primary ureteral transitional cell carcinoma after radical nephroureterectomy. J Urol 2009; 182: 451-8

13. Goonewardena S, Jayarajah U, Kuruppu S, Herath H, Fernando D, Vickneswaran K. Clinical outcomes in a cohort of patients with $\mathrm{T} 1$ high grade urothelial bladder cancer not receiving intravesical bacillus Calmette-Guerin: a 15 year experience. Ceylon Med J 2018; 63(1):37-39

14. Margulis V, Shariat SF, Matin SF, et al. Outcomes of radical nephroureterectomy: a series from the Upper Tract Urothelial Carcinoma Collaboration. Cancer 2009; 115: 1224-33. 